\title{
The effect of tea consumption on the steroid profile
}

Sergi Coll ${ }^{1,2}$, Xavier Matabosch ${ }^{1}$, Lorena Garrostas ${ }^{1}$, Nuria Monfort ${ }^{1}$, Clara PerezMaña $^{3,4}$, Nieves Pizarro ${ }^{3,4}$, Julián A Mateus ${ }^{3}$, Mohammed Ezzel ${ }^{3}$, Rafael de la Torre ${ }^{2,3,5}$, Rosa Ventura ${ }^{1,2}$

(1) Catalonian Anti-Doping Laboratory, Doping Control Research Group, IMIM, Hospital del Mar, Barcelona, Spain

(2) Department of Experimental and Health Sciences, Universitat Pompeu Fabra, Barcelona, Spain

(3) Integrated Pharmacology and Neurosciences Systems Research Group, IMIM, Barcelona, Spain

(4) Autonomous University of Barcelona, Bellaterra, Spain

(5) Spanish Biomedical Research Centre in Physiopathology of Obesity and Nutrition (CIBEROBN), Instituto Salud Carlos III, Madrid, Spain

Corresponding author: Rosa Ventura Alemany

Doctor Aiguader 88, 08003 Barcelona, Spain

E-mail: rventura@imim.es

Phone: +0034-933160471

Fax: +0034-933160499 


\section{Abstract}

Green tea (GT), along with its flavonol epigallocatechin-3-gallate (EGCG), has shown to inhibit the UGT2B17 isoenzyme, which is highly involved in the glucuronidation of testosterone (T) and its metabolites. Since the steroid profile (SP) is composed of urinary concentrations of $\mathrm{T}$ and related metabolites excreted in both the free and the glucuronide fractions, GT consumption could alter the SP leading to misunderstanding in doping controls. The aim of the present work was to study the effect of GT consumption on the SP.

This study was performed with 29 male volunteers, which could be classified in two arms depending on their T/E values $(0.12 \pm 0.02, \mathrm{n}=12 ; 1.64 \pm 0.90, \mathrm{n}=17)$. The clinical protocol was designed to evaluate the effect of GT administration on the SP biomarkers. Participants were asked to consume GT with a high content of EGCG for 7 days (5 GT beverages along the whole day for days 1-6 and 9 GT beverages in day 7, corresponding to 520 and $936 \mathrm{mg} /$ day of EGCG, respectively). Urine samples were collected before and during GT consumption at different time periods. The SP was measured using gas chromatography-mass spectrometry.

The excretion rates of the SP metabolites did not change after GT consumption. Moreover, the individual evaluation of the subject's steroidal biological passport resulted in normal sequences.

The results obtained show that GT consumption does not distort the establishment of normal ranges of SP parameters. Therefore, GT consumption does not need to be considered a confounding factor in the SP evaluation.

Key Words: Doping, EAAS, Green tea, Steroid profile, EGCG. 


\section{Introduction}

The misuse of synthetic endogenous anabolic androgenic steroids (EAAS) is the antidoping rule violation most detected in doping control analyses. The World Anti-Doping Agency (WADA) implemented the steroid profile (SP) as a part of the Athlete's Biological Passport (ABP) in order to detect suspicious samples of exogenous administration of EAAS. ${ }^{[1,2]}$ The ABP steroidal module consists of an individual and longitudinal monitoring of SP biomarkers to be more efficient detecting changes that can reveal the administration of EAAS. ${ }^{[3,4]}$ If changes are observed, the sample has to be confirmed by gas chromatography isotope ratio mass spectrometry (GC/C/IRMS) to demonstrate the exogenous origin of testosterone $(\mathrm{T})$ and related metabolites. ${ }^{[1]}$ The SP consists of the determination of concentrations of various endogenously produced steroidal hormones: T, epitestosterone (E), androsterone (A), etiocholanolone (Etio), $5 \alpha$ -

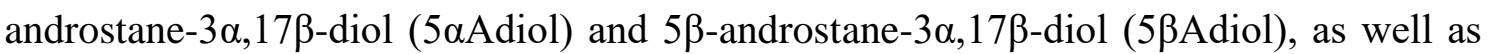

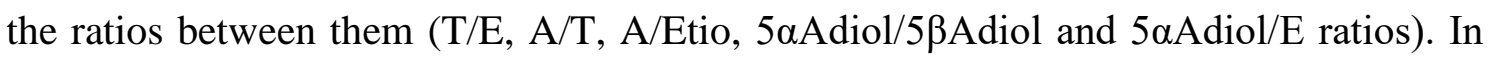
humans, androgen steroids are mainly excreted in urine as conjugates with glucuronic acid and, therefore, the SP is measured using both the free and the glucuronide fractions.

UDP-glucuronosyltransferases (UGTs) are the enzymes responsible for the catalysis of androgen conjugation with glucuronic acid. ${ }^{[5]}$ UGTs are divided into different subfamilies, ${ }^{[6]}$ and the most important enzymes involved in the glucuronidation of androgens belong to the subfamily UGT2B. ${ }^{[7,8]} \mathrm{T}$ glucuronidation is mainly dependent on UGT2B17 and to a lesser extent on UGT2B15. ${ }^{[7,9]}$ Importantly, UGT2B17 is not involved in E glucuronidation, the enzyme responsible for E glucuronidation seems to be UGT2B7, which has no activity on T glucuronidation. ${ }^{[9,10]} 5 \alpha$ Adiol, 53Adiol, A and Etio are also conjugated by UGT2B17 and by other isoenzymes. ${ }^{[11]}$ UGT2B7 conjugates all four androgens, exhibiting high glucuronidation rates towards steroids with $5 \alpha$ configuration (5 $\alpha$ Adiol and A), and UGT2B15 conjugates $5 \alpha$ Adiol and $5 \beta$ Adiol. $^{[11]}$

Genetic polymorphisms may alter the expression or the activity of UGTs. ${ }^{[12]}$ It is well known that a common deletion polymorphism occurs in the gene coding for the isoenzyme UGT2B17. This gene deletion results in less excretion of T glucuronide in urine in individuals homozygous for the UGT2B17 deletion ( $\mathrm{del} / \mathrm{del}$ ), compared to individuals with one (ins/del) or two (ins/ins) functional alleles of the UGT2B17 
gene. ${ }^{[13]}$ The UGT2B17 polymorphism also causes variations in the excretion of glucuronides of other T related metabolites. Homozygotes del/del are more prevalent in Asian than in Caucasian population, with rates of $66.7 \%$ and $9.3 \%$, respectively. ${ }^{[13]}$ It has important practical implications in doping controls because if a single cut-off value for T/E ratio was applied to the whole population, athletes with the gene deletion would have an unfair advantage in case of exogenous administration of T. Furthermore, gender differences in UGT2B17 expression and glucuronidation activity have been described. ${ }^{[14]}$ Therefore, the use of the subject-based testing approach of the ABP is of paramount importance.

In a previous study, using in vitro tests, it was shown that $\mathrm{T}$ glucuronidation by UGT2B17 isoenzyme is inhibited by green and white tea extracts along with flavonols from tea: epicatechin, epigallocatechin-3-gallate (EGCG) and catechin gallate. ${ }^{[15]}$ Due to the involvement of UGT2B17 in the glucuronidation of other androgens, ${ }^{[11]}$ it is expected that this inhibition will also affect the formation of glucuronides of other steroids included in the SP. In summary, the consumption of tea may produce alterations in the SP leading to misinterpretation on the longitudinal studies and/or masking the exogenous administration of some EAAS. Since tea is the most widely consumed beverage in the world next to water, it is of utmost importance to study the relevance of the UGT2B17 gene inhibition by tea constituents in the in vivo metabolism of all androgens included in the SP. If important alterations of the urinary SP are found, the monitoring of the markers of tea consumption will be needed when detecting an atypical profile before going to the expensive and time-consuming GC/C/IRMS analysis.

The aim of the present study was to investigate the effect of green tea (GT) administration on the SP biomarkers. For this purpose, healthy male individuals with a wide range of T/E ratio values were asked to consume GT with a high content of EGCG for 7 days, and the SP was measured. 


\section{Experimental}

\section{Clinical study}

A total of 29 healthy male volunteers (19 Caucasian and 10 Asian volunteers) were recruited to participate in the study (mean age $25 \pm 6$ years). Participants were divided into two groups according to their baseline T/E ratio values and their baseline urinary $\mathrm{T}$ excretion levels (Table 1). Participants included in arm 1 were individuals with low T/E ratio values, considered to present UGT2B17 gene deletion, and volunteers included in arm 2 were individuals with higher T/E ratio values, considered to have one or two functional alleles of the UGT2B17 gene. There were no significant differences in the average age, weight, and height among groups.

Participants were usual consumers of beverages containing methyl-xanthines in order to guarantee that they can tolerate the consumption of a large number of tea drinks. Participants were on a low catechin diet and were asked not to ingest drugs that could influence the SP during the study, including alcoholic beverages. Drugs of abuse urine tests (Instant-View, Multi-Panel 4 Test Drug Screen, Alfa Scientific Designs, Poway, CA, USA) and breath alcohol tests (Dräger Breathalyzer 7410 Plus, Denmark) had to be negative for inclusion in the study.

A clinical trial involving GT consumption and collection of urine samples was performed (protocol code IMIMFTCL/EGCG/3). Ethical approval of the study was granted by the local Research Ethical Committee (Comitè Ėtic d'Investigació Clínica CEIC-Parc de Salut Mar, Barcelona, Spain). All subjects participating in the study gave their written informed consent prior inclusion and underwent a general physical examination, routine laboratory test, urinalysis, and a 12-lead electrocardiogram with results within normal values.

The clinical protocol was designed to determine the effect of a repetitive GT consumption on the SP, exceeding a normal GT consumption. The content of EGCG, the main flavonoid of tea, is quite variable even for the same tea brand from country to country. In this study, a GT with a high content of EGCG was selected. ${ }^{[16]}$

The protocol of GT consumption as well as urine and blood collection periods are described in Table 2. In the pre-administration phase, volunteers were requested to refrain from tea intake $24 \mathrm{~h}$ before taking part in the study and baseline urine samples were collected for 3 consecutive days. In the intervention phase, urine samples were 
collected for 8 consecutive days and participants were asked to consume 5 tea beverages along the whole day for 6 consecutive days, and in day 7, they consumed 9 tea beverages. In total, $24 \mathrm{~h}$ total urine was collected during eleven days; the urine collection periods (UC) were different, depending on the experimental day, and they are described in Table 2. All urine samples were stored at $-20^{\circ} \mathrm{C}$ until analysis.

Blood samples were also collected before and after GT consumption (Table 2). Blood was collected just before first GT consumption in day 1, as well as at preadministration, and 1 and $2 \mathrm{~h}$ after first GT ingestion in day 7. Blood samples (4 mL) were collected in lithium heparin tubes and plasma was obtained by centrifugation (10 $\left.\min , 3000 \mathrm{rpm}, 4^{\circ} \mathrm{C}\right) .350 \mu \mathrm{L}$ plasma aliquots were transferred to low-binding tubes containing $20 \mu \mathrm{L}$ of a preservative solution $\left(0.4 \mathrm{M} \mathrm{NaH}_{2} \mathrm{PO}_{4} \cdot \mathrm{H}_{2} \mathrm{O}, 20 \%\right.$ ascorbic acid, $0.1 \%$ EDTA, $\mathrm{pH}=3.6$ ). Samples were immediately stored at $-80^{\circ} \mathrm{C}$ until analysis, which was performed not longer than 1 week after sample collection.

The GT beverage was prepared as follows: one tea bag was immersed for $15 \mathrm{~min}$ in a ceramic cup with a lid containing $200 \mathrm{~mL}$ of hot water. The water was previously heated in a microwave oven for $2 \mathrm{~min}$. Then, the tea bag was drained and removed. The GT was consumed within a time period no longer than 10 min after the tea bag was removed (no more than $25 \mathrm{~min}$ from the addition of the tea bag to water). When GT was prepared using two tea bags, the procedure was the same, but adding two tea bags instead.

\section{Chemicals and reagents}

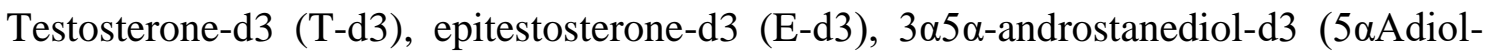
d3), 3 $\alpha 5 \beta$-androstanediol-d5 (5ßAdiol-d5), androsterone-d4 (A-d4), androsterone-d4glucuronide (A-d4-gluc), etiocholanolone-d5 (Etio-d5), methyltestosterone, testosterone (T), androsterone (A) and etiocholanolone (Etio) were purchased from the Australian National Measurement Institute (Pymble, Australia). Sodium hydrogen carbonate,

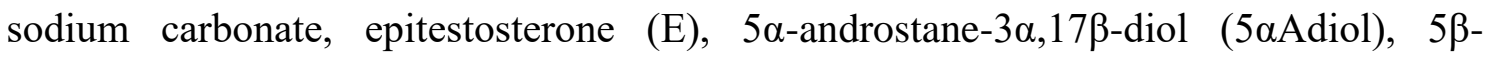
androstane-3 $\alpha, 17 \beta$-diol (5 $\beta$ Adiol) and ethylgallate were obtained from Sigma (St Louis, MO, USA). Epigallocatechin-3-gallate (EGCG) was purchased from Extrasynthese (Lyon, France). $\beta$-Glucuronidase from Escherichia coli K12 was provided by Roche Diagnostics GmbH (Mannheim, Germany). Disodium hydrogen phosphate, sodium 
hydrogen phosphate, tert-butyl methyl ether, ammonium iodide and 2-mercaptoethanol were supplied by Merck (Darmstadt, Germany). N-Methyl-Ntrimethylsilylfluoroacetamide (MSTFA) was purchased from Macherey-Nagel (Düren, Germany). Ultra-pure water was obtained using Milli-Q purification system (Millipore Ibérica, Barcelona, Spain). DetectabuseTM solid-phase extraction (SPE) columns were purchased from Biochemical Diagnostics Inc. (New York, NY, USA).

\section{Measurement of EGCG in the GT beverages}

EGCG content in the GT beverages was measured after preparing a GT infusion as described previously. Samples were diluted (1/10) with Milli-Q water and immediately analysed by liquid chromatography coupled to tandem mass spectrometry (LCMS/MS). Quantification was performed in an Agilent 1200 series LC system (Agilent Technologies) coupled to a triple-quadrupole (6410 Triple Quad LC-MS; Agilent) mass spectrometer with an electrospray interface. The chromatographic separation was performed using an Acquity UPLC BEH C18 (100 mm × $3.0 \mathrm{~mm}$ i.d., $1.7 \mu \mathrm{m})$ column. The mobile phase consisted of formic acid $0.1 \%$ (A) and acetonitrile with formic acid $0.1 \%$ (B). The gradient was: $0.5 \% \mathrm{~B}$, for $1 \mathrm{~min}$; to $50 \% \mathrm{~B}$, in $2 \mathrm{~min} ; 50 \% \mathrm{~B}$, for $5 \mathrm{~min}$; to $0.5 \% \mathrm{~B}$, in $0.2 \mathrm{~min} ; 0.5 \% \mathrm{~B}$, for $2.8 \mathrm{~min}$. The flow rate was $0.3 \mathrm{~mL} / \mathrm{min}$, and the injection volume was $5 \mu \mathrm{L}$. All compounds were monitored in positive ionization using the multiple reaction monitoring mode. Mass/charge values selected for identification of analytes were as follows: EGCG m/z 457 $\rightarrow 169$, 305, 331, fragmentor (F) $150 \mathrm{~V}$, collision energy (CE) $15 \mathrm{eV}$; and ethylgallate (internal standard) m/z 197 $\rightarrow 78,124$, 169, F $141 \mathrm{~V}, \mathrm{CE} 15 \mathrm{eV}$.

\section{Measurement of EGCG in plasma}

Determination of free EGCG concentration in plasma samples was performed by an extraction procedure described by Martí et al. ${ }^{[17]}$ Chromatographic analysis was performed as described in previous section.

\section{Analysis of the steroid profile in urine}


The SP was measured in $2.5 \mathrm{~mL}$ aliquots of urine by gas chromatography-mass spectrometry (GC-MS) using a previously described method. ${ }^{[18]}$ Briefly, after addition of $25 \mu \mathrm{L}$ of the internal standard solution $(5 \mu \mathrm{g} / \mathrm{mL}$ of $\mathrm{T}-\mathrm{d} 3$ and $\mathrm{E}-\mathrm{d} 3,10 \mu \mathrm{g} / \mathrm{mL}$ of 5 $\alpha$ Adiol-d3, 5ßAdiol-d5 and methyltestosterone, $19.2 \mu \mathrm{g} / \mathrm{mL}$ of A-d4-glucuronide, and $12 \mu \mathrm{g} / \mathrm{mL}$ of Etio-d5), urine samples were adjusted to $\mathrm{pH} 7$ with $1 \mathrm{~mL}$ of $1 \mathrm{M}$ phosphate buffer. Then, enzymatic hydrolysis was performed adding $30 \mu \mathrm{L}$ of $\beta$-glucuronidase enzyme from Escherichia coli. The mixture was incubated for $1 \mathrm{~h}$ at $55^{\circ} \mathrm{C}$ in a water bath. After incubation, samples were alkalinized with $200 \mathrm{mg}$ of a mixture of sodium hydrogen carbonate and sodium carbonate buffer $(1: 2, \mathrm{w} / \mathrm{w})$, and the analytes were extracted by liquid-liquid extraction using $6 \mathrm{~mL}$ of tert-butyl-methyl ether. The organic phases were evaporated to dryness under nitrogen stream, and the residue was derivatised with $50 \mu \mathrm{L}$ a mixture of MSTFA/ $\mathrm{NH}_{4} \mathrm{I} / 2$-mercaptoethanol (1000:2:6, v:w:v) for $20 \mathrm{~min}$ at $60^{\circ} \mathrm{C}$.

The extracts $(2 \mu \mathrm{L})$ were analysed by GC-MS using a 7890A GC connected to a 7693 autosampler and coupled to a 5975C single quadrupole MS (Agilent Technologies, Santa Clara, CA, USA). The GC was equipped with a capillary column HP-Ultra1 (16.5m x $0.2 \mathrm{~mm}$ inner diameter with a $0.11 \mu \mathrm{m}$ film thickness) from $\mathrm{J} \& \mathrm{~W}$ Scientific (Folsom, CA, USA). The GC temperature was ramped as follows: initial temperature $180^{\circ} \mathrm{C}$, increased at $3{ }^{\circ} \mathrm{C} / \mathrm{min}$ to $230^{\circ} \mathrm{C}$, then at $40^{\circ} \mathrm{C} / \mathrm{min}$ to $310^{\circ} \mathrm{C}$ and maintained at the final temperature for $3 \mathrm{~min}$. The injector and transfer lines were kept at $280^{\circ} \mathrm{C}$. A split ratio 1:20 was used. Helium was used as a carrier gas working under constant pressure (13.9 psi). The electron ionization source was set at $230^{\circ} \mathrm{C}$. The MS was operated in electron ionization mode $\left(70 \mathrm{eV}, 150{ }^{\circ} \mathrm{C}\right)$, and in selected ion monitoring acquisition mode. The performance characteristics of the method have been previously described. ${ }^{[17]}$ The quality of the analysis was controlled by quality control urine samples containing low and high representative concentrations of the markers of the SP.

\section{Data analysis}

The parameters of the SP were measured in all urine samples. The ratios A-mono-OTMS/A-bis-O-TMS and And-d4/Etio-d5 were measured in order to verify the percentages of derivatization and hydrolysis, respectively. Additionally, the ratios $5 \alpha-$ androstandione/A and $5 \beta$-androstandione/Etio ratios were measured in order to evaluate 
the microbial degradation, and ensure that the SP was valid according to WADA criteria. ${ }^{[1]} \mathrm{SP}$ data from the entire population of volunteers was analysed using the ABP software provided by WADA. It provides the longitudinal profiles of the T/E, A/T,

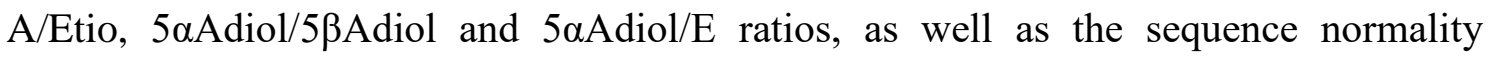
expressed as a percentage (specificity set at 99\%) and the doping prevalence. Urine samples collected before GT administration were used to establish baselines ratios, and each post-administration sample was independently analysed against the baseline to determine any atypical finding (suspicious result in which further investigation should be conducted). An out-of-range value was defined as a ratio value exceeding the individual threshold, resulting in an atypical profile.

Statistical analyses were performed in order to study metabolite excretion differences between pre-administration samples and urines collected in the administration phase. Daily excretion profiles were compared using analysis of variance of repeated measures (ANOVA) followed by a Bonferroni adjusted t test. Wilcoxon Sign test was used to assess if SP components exhibit variations in their excretion amounts over the day. To study plasmatic EGCG levels, we used Student's two-tailed $t$ test. Statistical analyses were performed using the computer software SPSS 12.0 (SPSS Inc., Chicago, IL, USA), and the level of significance was established at $p<0.05$ in all tests. 


\section{Results and discussion}

The effect of GT consumption on the SP was assessed in healthy male volunteers covering a broad range of T/E ratio values (Table 1). Arm 1, included 12 participants (4 Caucasian and 8 Asian volunteers); and arm 2, included 17 volunteers (15 Caucasian and 2 Asian volunteers) (Table 1). One of the subjects included in arm 2 showed a low basal T/E ratio $(0.47 \pm 0.07)$, but he was allocated in arm 2 because he presented a basal $\mathrm{T}$ excretion of $25 \pm 5 \mathrm{ng} / \mathrm{min}$, more similar to the profiles of the volunteers considered to have one or two functional alleles of the UGT2B17 gene. This distribution of volunteers is in accordance with a previous study, where a prevalence of the absence of the UGT2B17 gene seven times more common in Asian than Caucasian population is described. ${ }^{[13]}$

Despite the fact that participants were asked not to take any of the drugs that are known to alter the $\mathrm{SP},{ }^{[19-21]}$ the presence of ethyl glucuronide (metabolite of ethanol) was detected in urine samples collected in days 6,7 and 8 of a participant belonging to arm 2. Consequently, statistical evaluation was carried out just with data obtained till day 5. Moreover, 3 volunteers belonging to arm 2 did not perform sample collection completely and, for this reason, the statistical evaluation could not be performed with all their data.

\section{EGCG content in GT beverages}

The content of EGCG in the tea bags was measured in order to verify the amount of EGCG administered. The mean content of EGCG in tea bags from 7 different lots was $103.8 \pm 8 \mathrm{mg} /$ tea bag (range 90.8-117.5 mg/tea bag). These results are in accordance with published data. ${ }^{[16]}$ EGCG content variations of the same order were obtained when measuring tea bags from the same lot. Therefore, the consumption of 5 and 9 tea beverages is equivalent to $520 \mathrm{mg}$ and $936 \mathrm{mg}$ of EGCG, respectively.

\section{EGCG concentrations in plasma}

Plasma concentration of free EGCG was measured in order to confirm its absorption (Figure 1). Plasma concentrations of free EGCG obtained are consistent with results 
previously described. ${ }^{[22]}$ EGCG was not detected in plasma before GT administration in day 1 , but measurable concentrations were detected before and after GT consumption (2 GT bags; $168 \mathrm{mg}$ of EGCG) in day 7. Participants from both arms showed measurable concentrations of EGCG in most of the pre-administration plasma samples obtained in day 7, meaning that small amounts are accumulated after repeated doses during 6 days. These findings are in agreement with reported EGCG pharmacokinetics. ${ }^{[22]}$ For the two treatment groups, EGCG concentrations determined 1h after GT administration were higher than those obtained $2 \mathrm{~h}$ after GT ingestion, but the differences did not reach statistical significance.

Measured plasma concentrations of free EGCG ranged from 0.2 to $720 \mathrm{nM}$. In a previous study, using in vitro tests, it was demonstrated that EGCG inhibits the glucuronidation of $\mathrm{T}$ by the enzyme UGT2B17 with a $\mathrm{IC}_{50}$ inhibition value of 65000 $n M^{[15]}$ Therefore, the results indicate that EGCG plasma concentrations are much lower than the concentration described to inhibit the UGT2B17 enzyme not only hours after consuming GT, but also after consuming large amounts of GT for 6 consecutive days. On the other hand, statistically significant lower plasma concentrations of EGCG were observed in subjects of arm 2 compared with subjects of arm 1. This result might be related to different capacity of glucuronoconjugation of EGCG. Subjects of arm 1, with lower capacity of glucuronoconjugation, have higher levels of free EGCG than subjects of arm 2. The fact that EGCG metabolism seems to be dependent on UGT2B17 enzymatic activity presence could explain why Asian population displaying lower activities presents a lower prevalence for some chronic diseases, such as diabetes, cardiovascular diseases and prostate cancer. ${ }^{[23-26]}$

\section{Steroid metabolites excretion}

Concentrations of the metabolites monitored in the SP were measured in all collected samples. The effect of GT consumption was assessed by evaluating the $24 \mathrm{~h}$ excretion rates as well as excretion rates obtained along the day.

- $24 \mathrm{~h}$ excretion rates

The effect of GT consumption was assessed by evaluating $24 \mathrm{~h}$ excretion rates because EAAS metabolism is based on a daily homeostatic balance. ${ }^{[27]}$ Steroid amounts of the 
urines collected at different time fractions were combined to obtain $24 \mathrm{~h}$ excretion rates. In Figure 2, 24h excretion rates of SP metabolites obtained during the whole study are presented for volunteers of arm 1 and arm 2. As can be seen, excretion rates for all metabolites were constant over the 11 days of the study (pre-administration and intervention phases) for both groups of volunteers. No statistically significant changes were observed during the first 6 days of the administration phase when 5 GT beverages were consumed, not even after the consumption of 9 GT beverages in day 7 . No significant alterations were either observed in day 8 , when no tea was consumed. Noticeable outliers in 5 $\beta$ Adiol daily excretions were presented by one volunteer included in arm 2. This volunteer did not collect all the requested urines, and daily excretion amounts could not be calculated in days -3, 1, 6 and 8. For this reason, outliers are not shown in these days.

Due to the inhibition of the enzyme UGT2B17 by tea components, ${ }^{[15]}$ it was expected that mainly individuals with functional alleles of the UGT2B17 gene (arm 2) present variations in their steroid excretion levels after GT consumption: important alterations were expected to take place in $\mathrm{T}$ excretion and, in lesser extent, in the excretion of other SP metabolites. However, our results demonstrate that GT consumption has no effect on the excretion rates of the metabolites, even at high doses, in neither of the two groups (arm 1 and arm 2).

The clinical study only included male volunteers. Since men present a higher UGT2B17 glucuronidation activity than women, ${ }^{[14]}$ the potential gene inhibition by EGCG is expected to have a lower impact in females. Therefore, after observing that EGCG has not effect on SP metabolites excretion in men, the same unnoticeable effect is expected in women.

- Excretion rates within a day

Variations in EAAS urinary concentrations along the day have been previously reported, ${ }^{[28]}$ and these fluctuations are considered to be the normal intraindividual variation. In order to evaluate the potential effect of GT along the day, data from urines collected in days $-2,-1,1,7$, and 8 (UC2 in Table 2) were compared. In day 1, a potential immediate GT effect was evaluated, and data from urines collected in day 7 
were used to assess the effect of the administration of the maximum dose. The excretion profiles are presented in Figure 3.

No excretion differences were observed over the course of the day in response to GT administration for any of the SP metabolites. No differences were either observed when comparing data from each day with each time fraction.

The excretion rates obtained in different time fractions from day 1 or day 7 were statistically compared with those obtained in day -2 and day -1 (Table 1 , Supplementary information). A change was considered significant if differences were statistically significant when comparing each point with the corresponding point from both basal days. A significant change was observed only in one point for $5 \alpha$ Adiol and in a different point for $5 \beta$ Adiol. Since these changes did not follow a clear pattern, they were not considered as a robust enough, and for this reason, it is concluded that the SP metabolites did not show excretion changes along the day after GT consumption.

\section{Longitudinal evaluation of steroid profiles}

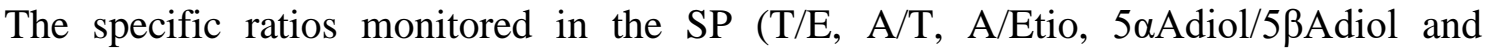
$5 \alpha$ Adiol/E) were evaluated in all collected urines. Taking into account that previous longitudinal studies demonstrated that T/E variations remain within $30 \%$ of the individual mean for males, ${ }^{[29]}$ no important changes were observed in the T/E ratios before and after GT consumption for all volunteers participating in both groups. The

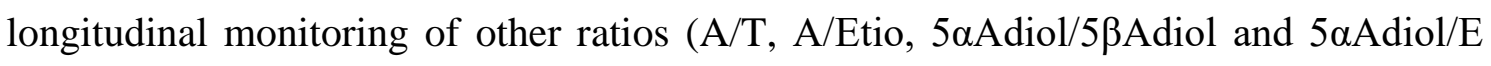
ratios) did not lead to any abnormality. Moreover, means and standard deviations of each monitored ratio were constant along the whole study for both arms (Table 3). The differences observed between the ratios of the two arms are already described.

Although the initial analyses consisting of evaluating excretions and ratios did not result in any suspicious SP, the individual application of the ABP program is the most sensitive screening method. ${ }^{[30]}$ Therefore, the ABP statistic model was applied to the data of all subjects. The data from 10 urine samples collected in the pre-administration phase (days -3, -2 and -1) was used to establish reference ABP values for each volunteer. Then, the 22 urine samples collected in the intervention phase were individually compared with the baseline levels established by the ABP program. It is 
worth to notice that samples collected during the pre-administration phase resulted in normal ABP sequences.

After the ABP evaluation, no outliers and no atypical profiles were obtained for the T/E ratio of any of the participants during the administration phase. As an example, Figure 4 shows normal sequences of the T/E ratio after the application of the $\mathrm{ABP}$ model in one Asian and one Caucasian volunteer from arm 1 and arm 2, respectively. However, two subjects from arm 2 triggered atypical high $5 \alpha$ Adiol/5 $\beta$ Adiol ratios in few of their samples. In a similar study performed by our group, ${ }^{[18]}$ the $5 \alpha$ Adiol/5 $\beta$ Adiol ratio was also the only one that led to few abnormal high sequences, and they were not considered to be suspicious profiles because it is known that this ratio has confounding factors. ${ }^{[19]}$ As observed in our previous study, samples leading to an abnormal $5 \alpha$ Adiol/5 $\beta$ Adiol sequence did not meet the WADA-stipulated positivity criteria for $5 \alpha$ Adiol and 5ßAdiol. ${ }^{[1]}$ Moreover, these two volunteers flagged normal profiles for T/E, A/T and $5 \alpha$ Adiol/E ratios, and also presented a constant 5 $\beta$ Adiol excretion during the whole study. For these reasons, these atypical sequences were not considered to be caused by GT consumption. 


\section{Conclusion}

In conclusion, GT consumption did not lead to changes in the excretion rates of $\mathrm{T}$ and the other SP metabolites. Excretion rates were constant both within the same day and in daily periods during GT administration. Therefore, our results indicate that despite the fact that in vitro tests showed that catechins inhibit glucuronidation activity of UGT2B17, the administration of a large amount of GT, which highly exceeds a standard GT consumption, did not result in plasma concentrations of EGCG high enough to inhibit in vivo UGT2B17 activity. Moreover, it is also concluded that catechins from GT do not have an in vivo effect on other UGT2B isoenzymes. Finally, the steroidal ABP evaluation of the entire population of participants resulted in normal sequences, showing no differences between groups. For this reason, GT cannot be considered as a confounding factor of the steroid module of the ABP and it is not necessary to take GT consumption into consideration for the SP evaluation.

The negative impact of GT consumption on the steroidal module of the ABP enforces the use of the SP for the detection of EAAS misuse. 


\section{Acknowledgements}

The authors are grateful for the financial support received from the WADA (research grants 13D26RV). This work has been carried out with the support of ConsellCatalà de l'Esport (Generalitat de Catalunya). Authors thank Sergio Peña, Silvia Petit, Esther Menoyo, Marta Pérez and Soraya Martín for their collaboration in the excretion studies and management of samples, and Cristina Llop for assistance in the preparation of the clinical trial protocol.

\section{Conflict of interest}

The authors wish to disclose that there were no conflicts of interest associated with professional relationships, and that the results of the study are presented clearly, honestly, and without fabrication, falsification, or inappropriate data manipulation. 


\section{References}

1. WADA. Technical document for Endogenous Anabolic Androgenic Steroids. https://www.wada-ama.org/sites/default/files/resources/files/wada-td2016eaas-eaasmeasurement-and-reporting-en.pdf. Accessed December 13, 2017.

2. WADA. Guidelines for Athlete Biological Passport (ABP). https://www.wadaama.org/sites/default/files/resources/files/guidelines_abp_v6_2017_jan_en_final.pdf. Accessed December 13, 2017.

3. Ayotte C. Detecting the administration of endogenous anabolic androgenic steroids. In: Thieme D, Hemmersbach P, ed. Doping in Sports, Handbook of Experimental Pharmacology. 1st ed. Berlin: Springer, Inc; 2010:77-98.

4. Sottas PE, Robinson N, Saugy M. The athlete's biological passport and indirect markers of blood doping. In: Thieme D, Hemmersbach P, ed. Doping in Sports, Handbook of Experimental Pharmacology.1st ed. Berlin: Springer, Inc; 2010:305-326.

5. Kuuranne T. Phase-II metabolism of androgens and its relevance for doping control analysis. In: Thieme D, Hemmersbach P, ed. Doping in Sports, Handbook of Experimental Pharmacology.1st ed. Berlin: Springer, Inc; 2010:65-75.

6. Mackenzi PI, Owens IS, Burchell B, et al. The UDP glucosyltransferase gene superfamily: recommended nomenclature update based on evolutionary divergence. Pharmacogenetics 1997;7(4):255-269.

7. Turgeon D, Carrier JS, Lévesque E, Hum DW, Bélanger A. Relative enzymatic activity, protein stability, and tissue distribution of human steroid-metabolizing UGT2B subfamily members. Endocrinology 2001;142(2): 778-787.

8. Barbier O, Bélanger A. The cynomolgus monkey (Macacafascicularis) is the best animal model for the study of steroid glucuronidation. J Steroid Biochem 2003;85(25):235-245.

9. Sten T, Bichlmaier I, Kuuranne T, Leinonen A, Yli-Kauhaluoma Y, Finel M. UDPGlucuronosyltransferases (UGTs) 2B7 and UGT2B17 display converse specificity in testosterone and epitestosterone glucuronidation, whereas UGT2A1 conjugates with androgens similarly. Drug Metab Dispos 2009;37(2):417-423. 
10. Coffmann BL, King CD, Rios GR, Tephly TR. Glucuronidation of opioids, other xenobiotics, and androgens by human UGT2B7Y(268) and UGT2B7H(268). Drug Metab Dispos 1997;26(1):73-77.

11. Sten T, Kurkela M, Kuuranne $\mathrm{T}$, Leinonen A, Finel M. UDPGlucuronosyltransferases in conjugation of $5 \alpha$ - and $5 \beta$-androstane steroids. Drug Metab Dispos 2009;37(11):2221-2227.

12. Guillemette C, Lévesque E, Harvey M, Bellemare J, Menard V. UGT genomic diversity: beyond gene duplication. Drug Metab Rev 2010;42(1):24-44.

13. Jakobsson J, Ekström L, Inotsume N, et al. Large differences in testosterone excretion in Korean and Swedish men are strongly associated with a UDP-glucuronosyl transferase 2B17 polymorphism. J Clin Endocr Metab 2006;91(2):687-693.

14. Gallagher CJ, Balliet RM, Sun D, Chen G, Lazarus P. Sex differences in UDPGlucuronosyltransferase 2B17 expresion and activity. Drug Metab Dispos 2010;38(12):2204-2209.

15. Jenkinson C, Petroczi A, Barker J, Naughton DP. Dietary Green and White teas suppress UDP-glucuronosyltransferase UGT2B17 mediated testosterone glucuronidation. Steroids 2012;77(6):691-695.

16. Henning SM, Fajardo-Lira C, Lee HW, Youssefian AA, W Go VL, Heber D. Catechin content of 18 teas and green tea extract supplement correlates with the antioxidant capacity. Nutr Cancer 2003;45(2):226-235.

17. Martí MP, Pantaleón A, Rozek A, et al. Rapid analysis of procyanidins and anthocyanins in plasma by microelution SPE and ultra-HPLC. J Sep Sci 2010;33(1718):2841-2853.

18. Coll S, Matabosch X, Garrostas L, Perez-Maña C, Ventura R. Effect of glucocorticoid administration on the steroid profile. Drug Test Anal 2018;1-9.

19. Mareck U, Geyer H, Opfermann G, Thevis M, Schanzer W. Factors influencing the steroid profile in doping control analysis. J Mass Spectrom 2008;43(7):877-891. 
20. Kuuranne T, Saugy M, Baume N. Confounding factors and genetic polymorphism in the evaluation of individual steroid profiling. Br J Sports Med 2014;48(10):848-855.

21. Grosse J, Anielski P, Sachs H, Thieme D. Ethylglucuronide as a potential marker for alcohol-induced elevation of urinary testosterone/epitestosterone ratios. Drug Test Anal 2009;1(11-12):526-530.

22. Sherry Chow HH, Cai Y, Hakim IA, et al. Pharmacokinetics and safety of green tea polyphenols after multiple-dose administration of epigallocatechin gallate and polyphenon E in healthy individuals. Clin Cancer Res 2003;9(9):3312-3319.

23. Iso $\mathrm{H}$, Date $\mathrm{C}$, Wakai $\mathrm{K}$, et al. The relationship between green tea and total caffeine intake and risk for self-reported type 2 diabetes among Japanese adults. Ann Intern Med 2006;144(8):554-562.

24. Wu CH, Lu FH, Chang CS, Chang TC, Wang RH, Chang CJ. Relationship among habitual tea consumption, percent body fat, and body fat distribution. Obes Res 2003;11(9):1088-1095.

25. Sumpio BE, Cordova AC, Berke-Schlessel DW, Qin F, Chen QH. Green tea, the "Asian paradox," and cardiovascular disease. J Am Coll Surg 2006;202(5):813-825.

26. Lee PMY, Ng CF, Liu ZM, Ho WM, Lee MK, Wang F, Kan HD, He YH, Ng SSM, Wong SYS, Tse LA. Reduced prostate cancer risk with green tea and epigallocatechin 3-gallate intake among Hong Kong Chinese men. Prostate Cancer Prostatic Dis 2017;20(3):318-322.

27. Shackleton $\mathrm{CH}$. Profiling steroid hormones and urinary steroids. J Chromatogr 1986;379(1):91-156.

28. Mazzarino M, Bragano MC, de la Torre X, Molaioni F, Botrè F. Relevance of the selective oestrogen receptor modulators tamoxifen, toremifene and clomiphene in doping field: endogenous steroids urinary profile after multiple oral doses. Steroids 2011;76(12):1400-1406. 
29. Catlin DH, Hatton CK, Starcevic SH. Issues in detecting abuse of xenobiotic anabolic steroids and testosterone by analysis of athletes' urine. Clin Chem 1997; 43(7):1280-1288.

30. Miller GD, Nair V, Morrison MS, Summers M, Willick SE, Eichner D. Intranasal derlivery of Natesto®testosterone gel and its effects on doping markers. Drug Test Anal 2016;8(11-12):1197-1203. 
Table 1. Study population characteristics

\begin{tabular}{ccccccccc} 
arm & T/E ratio & $\begin{array}{c}\mathrm{T} \\
(\mathrm{ng} / \mathrm{min})\end{array}$ & $\mathrm{n}$ & $\begin{array}{c}\text { Caucasian } \\
\%(\mathrm{n})\end{array}$ & $\begin{array}{c}\text { Asian } \\
\%(\mathrm{n})\end{array}$ & $\begin{array}{c}\text { Height } \\
(\mathrm{cm})\end{array}$ & $\begin{array}{c}\text { Weight } \\
(\mathrm{kg})\end{array}$ & $\begin{array}{c}\text { BMI } \\
\left(\mathrm{kg} / \mathrm{m}^{2}\right)\end{array}$ \\
\hline 1 & $0.12(0.05-0.39)$ & $3 \pm 1$ & 12 & $33(4)$ & $67(8)$ & $177 \pm 4$ & $75 \pm 10$ & $24 \pm 3$ \\
\hline 2 & $1.54(0.40-5.43)$ & $42 \pm 26$ & 17 & $88(15)$ & $12(2)$ & $174 \pm 7$ & $68 \pm 17$ & $24 \pm 3$
\end{tabular}

Table 2. Clinical protocol: GT beverages consumed and collection of urine and blood samples. Urine sample collections: UC1, 0-15h and 15-24h; UC2, 0-5h, 5-10h, 10-15h and 15-24h. Blood samples collection: BC1, before the first GT consumption; BC2, before and 1 and $2 \mathrm{~h}$ after the first GT consumption.

\begin{tabular}{|c|c|c|c|c|c|c|c|}
\hline Days & -3 & -2 & -1 & 1 & $2-6$ & 7 & 8 \\
\hline Tea beverages & - & - & - & $5^{\mathrm{a}}$ & $5^{\mathrm{a}}$ & $9^{b}$ & - \\
\hline Urine collection & $\mathrm{UC1}$ & $\mathrm{UC2}$ & $\mathrm{UC2}$ & $\mathrm{UC2}$ & $\mathrm{UC1}$ & $\mathrm{UC2}$ & $\mathrm{UC2}$ \\
\hline Blood collection & - & - & - & $\mathrm{BC} 1$ & - & $\mathrm{BC} 2$ & - \\
\hline
\end{tabular}

Table 3. Means \pm SD values obtained after the longitudinal follow-up of SP ratios. Basal data comes from the mean of values obtained in days $-3,-2$ and -1 .

\begin{tabular}{|c|c|c|c|c|c|c|c|c|c|c|}
\hline & arm & Basal & Day 1 & Day 2 & Day 3 & Day 4 & Day 5 & Day 6 & Day 7 & Day 8 \\
\hline \multirow{2}{*}{$\mathrm{T} / \mathrm{E}$} & 1 & $0.12 \pm 0.07$ & $0.13 \pm 0.08$ & $0.12 \pm 0.07$ & $0.12 \pm 0.07$ & $0.13 \pm 0.08$ & $0.12 \pm 0.07$ & $0.12 \pm 0.07$ & $0.12 \pm 0.08$ & $0.12 \pm 0.07$ \\
\hline & 2 & $1.6 \pm 0.9$ & $1.7 \pm 1.0$ & $1.6 \pm 0.8$ & $1.7 \pm 1.0$ & $1.7 \pm 1.0$ & $1.7 \pm 1.1$ & $1.7 \pm 1.1$ & $1.7 \pm 0.9$ & $1.7 \pm 1.2$ \\
\hline $5 \alpha$ Adiol/ & 1 & $1.0 \pm 0.3$ & $1.1 \pm 0.3$ & $1.0 \pm 0.3$ & $1.1 \pm 0.3$ & $1.2 \pm 0.3$ & $1.1 \pm 0.4$ & $1.1 \pm 0.3$ & $1.2 \pm 0.4$ & $1.2 \pm 0.3$ \\
\hline $5 \beta$ Adiol & 2 & $0.5 \pm 0.3$ & $0.6 \pm 0.4$ & $0.5 \pm 0.4$ & $0.6 \pm 0.4$ & $0.5 \pm 0.4$ & $0.6 \pm 0.4$ & $0.5 \pm 0.3$ & $0.5 \pm 0.4$ & $0.5 \pm 0.3$ \\
\hline \multirow{2}{*}{$\mathrm{A} / \mathrm{T}$} & 1 & $596 \pm 426$ & $640 \pm 442$ & $574 \pm 368$ & $590 \pm 396$ & $497 \pm 286$ & $596 \pm 538$ & $555 \pm 410$ & $592 \pm 346$ & $648 \pm 422$ \\
\hline & 2 & $77 \pm 56$ & $79 \pm 63$ & $72 \pm 61$ & $79 \pm 69$ & $75 \pm 67$ & $70 \pm 53$ & $71 \pm 51$ & $78 \pm 59$ & $73 \pm 53$ \\
\hline \multirow{2}{*}{ A/Etio } & 1 & $1.3 \pm 0.5$ & $1.4 \pm 0.5$ & $1.4 \pm 0.5$ & $1.4 \pm 0.5$ & $1.3 \pm 0.5$ & $1.4 \pm 0.5$ & $1.4 \pm 0.6$ & $1.4 \pm 0.6$ & $1.5 \pm 0.6$ \\
\hline & 2 & $1.5 \pm 0.6$ & $1.6 \pm 0.7$ & $1.4 \pm 0.6$ & $1.5 \pm 0.6$ & $1.5 \pm 0.6$ & $1.6 \pm 0.7$ & $1.6 \pm 0.7$ & $1.5 \pm 0.7$ & $1.5 \pm 0.6$ \\
\hline \multirow{2}{*}{$5 \alpha$ Adiol/E } & 1 & $1.7 \pm 0.7$ & $1.9 \pm 0.9$ & $1.7 \pm 0.8$ & $1.7 \pm 0.8$ & $1.8 \pm 0.9$ & $1.8 \pm 1.0$ & $1.6 \pm 0.8$ & $1.7 \pm 0.9$ & $1.8 \pm 0.9$ \\
\hline & 2 & $2.3 \pm 1.1$ & $2.3 \pm 1.2$ & $2.2 \pm 1.1$ & $2.2 \pm 1.1$ & $2.2 \pm 1.0$ & $2.1 \pm 1.0$ & $2.1 \pm 0.9$ & $2.2 \pm 1.0$ & $2.2 \pm 1.2$ \\
\hline
\end{tabular}

